\title{
Many-body Green's function study of coumarins for dye-sensitized solar cells
}

\author{
C. Faber, ${ }^{1}$ I. Duchemin ${ }^{2}$, T. Deutsch ${ }^{2}$, X. Blase $^{1}$ \\ ${ }^{1}$ Institut Néel, CNRS and Université Joseph Fourier, B.P. 166, 38042 Grenoble Cedex 09, France. \\ ${ }^{2}$ INAC, SP2M/L_sim, CEA Cedex 09, 38054 Grenoble, France.
}

(Dated: October 31, 2018)

\begin{abstract}
We study within the many-body Green's function $G W$ and Bethe-Salpeter formalisms the excitation energies of several coumarin dyes proposed as an efficient alternative to ruthenium complexes for dye-sensitized solar cells. Due to their internal donor-acceptor structure, these chromophores present low-lying excitations showing a strong intramolecular charge-transfer character. We show that combining $G W$ and Bethe-Salpeter calculations leads to charge-transfer excitation energies and oscillator strengths in excellent agreement with reference range-separated functional studies or coupled-cluster calculations. The present results confirm the ability of this family of approaches to describe accurately Frenkel and charge-transfer photo-excitations in both extended and finite size systems without any system-dependent adjustable parameter, paving the way to the study of dye-sensitized semiconducting surfaces.
\end{abstract}

PACS numbers: 71.15.Qe,78.67.-n,78.40.Me,72.40.+w

\section{INTRODUCTION}

Promising to become a low-cost alternative to standard silicon-based photovoltaics, dye-sensitized solar cells (DSSC) have been intensively studied over the past 20 years $\stackrel{1.2}{1}$ The most prominent modern DSSCs, the socalled Grätzel cells, consist of porous layers of titanium dioxide nanoparticles covered by molecular dyes that absorb sun light. While most efficient sensitizers are composed of ruthenium dye complexes, intense research is conducted so as to find molecular alternatives which are cheaper, easier to synthesize, and free from the resource limitations related to the noble metal ruthenium. As a promising direction, Hara and coworkers ${ }^{3}$ demonstrated that coumarin-based dyes, such as the so-called NKX2xxx family (see Fig. 1), could lead to conversion efficiencies approaching that of ruthenium-based DSSCs.

Starting from the originally tested C343 coumarin 4 (Fig. [1 a), the introduction of (-C=C-) methine fragments between the coumarin unit and the terminal $(-\mathrm{COOH})$ carboxyl group (Fig. 2) induces a red shift of the absorption spectrum with improved light harvesting in the visible range,$\underline{3}$ The resulting molecular structures are represented in Figs. 1(b-d) showing the so-called (cis) conformations, with (trans) structural isomers represented in Fig. 2 for one of them. Further, inclusion of the cyano $(-\mathrm{C}=\mathrm{N})$ group enhances the acceptor character of the combined $(-\mathrm{COOH})$ and $(-\mathrm{C}=\mathrm{N})$ cyanoacrylic acid group, increasing the charge-transfer $(\mathrm{CT})$ character of the internal excitations, a factor believed to favor the injection of the photoelectron to the inorganic structure through the anchoring $(-\mathrm{COOH})$ carboxylic unit $\underline{3}$ Finally, the replacement of the methine spacer by thiophene chains ${ }^{5}$ reduces the adverse aggregation of dyes onto the $\mathrm{TiO}_{2}$ surface, leading to the NKX-2677 dye (Fig. 1e) with a solar-energy-to-electricity conversion efficiency of $7.7 \%$.

Due to its donor-acceptor structure, this family of molecules became also a benchmark for theoretical studies aiming at solving the well-known problem of describ- ing CT excitations within time-dependent density functional theory (TDDFT) $\underline{\underline{6} \cdot \underline{7}}$ Such difficulties arise from the lack of long-range electron-hole interaction when (semi)local kernels are used, as a signature of the missing non-local (screened) exchange contribution $\stackrel{8.9}{=}$ In particular, TDDFT calculations with local,,$\underline{10}$ hybrid $\underline{11,12}$ and range-separated hybrid (RSH) $\stackrel{11}{\underline{13}}$ kernels were conducted and compared to reference quantum chemistry coupled-cluster $\mathrm{CC} 2$ calculations $\underline{11}$ in order to assess the accuracy of the various approaches. The central role of CT excitations in organic and hybrid solar cells 14 certainly explains such efforts to develop computational techniques able to describe both Frenkel and CT excitations in a large variety of finite and extended systems with various screening environments.

In a few recent studies, many-body Green's function techniques, namely the so-called $G W^{15-21}$ and Bethe-Salpeter (BSE) $19,20,22-26$ formalisms, have been used to investigate the optical excitations in a family of small donor/acceptor organic dyads $\frac{27-29}{2}$ for which gas phase experiments ${ }^{30}$ are available. The low-lying $\mathrm{CT}$ excitations were found to come within $0.1 \mathrm{eV}$ as compared to experiment $\stackrel{28,29}{29}$ Besides these systems for which experimental data exist, optical excitations in a paradigmatic fullerene/thiophene derivative accep-

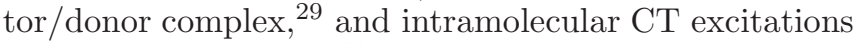
in a model dipeptide, $\stackrel{31}{=}$ have also been studied within the BSE formalism. This family of Green's function many-body techniques has been applied since the mideighties $17,18,21$ at the $a b$ initio level to study extended semiconductors and metals, but its applicability to the crucial problem of CT excitations in organic systems remains in its infancy and extensive tests and studies are still needed to rationalize its merits and limitations.

In the present work, we analyze the (singlet) excitation energies of various coumarin-based molecules such as the parent C343 dye and the related NKX-2388, NKX-2311, NKX-2586, and NKX-2677 structures for which reference calculations are available. We demonstrate that the 
a) $\mathrm{C} 343$

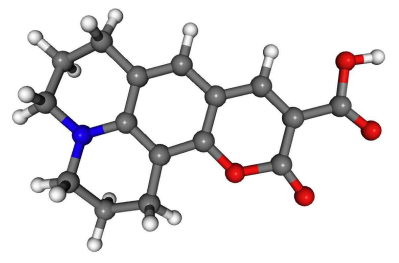

b) $\mathrm{Nkx2388} \mathrm{(cis)}$

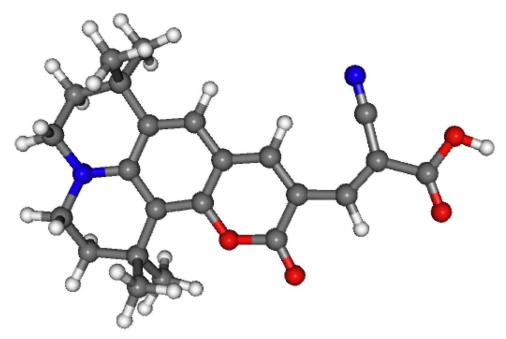

c) Nkx2311 (cis)

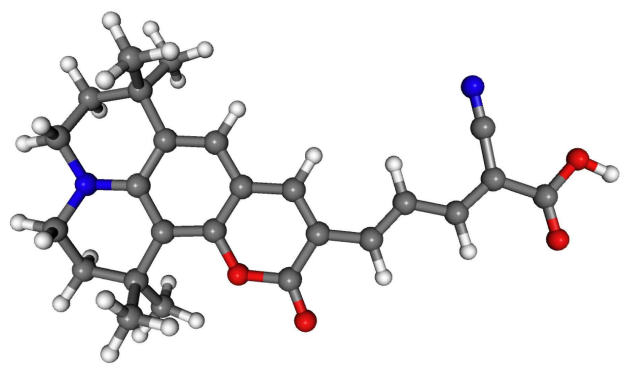

d) Nkx2586 (cis)

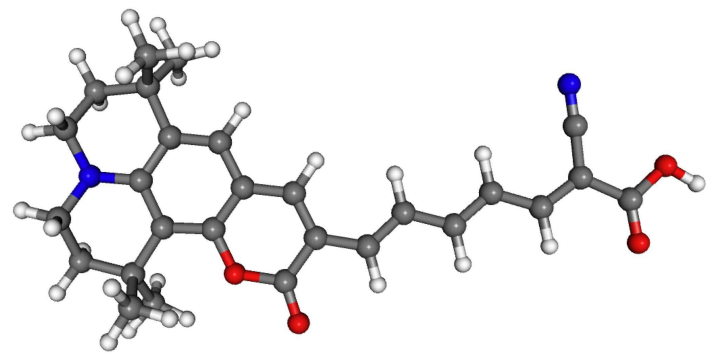

e) Nkx2677

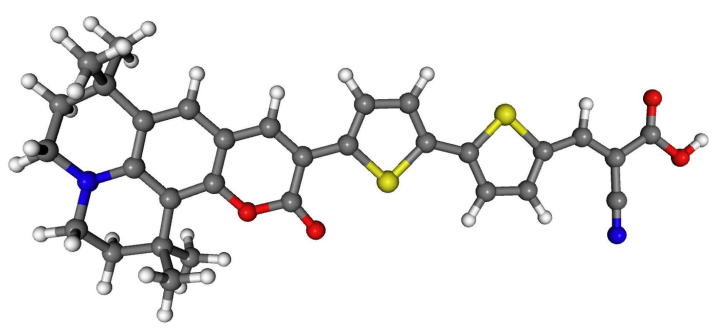

FIG. 1: (Color online) Symbolic representation of the studied coumarins: (a) parent C343, (b) NKX-2388 (cis), (c) NKX2311 (cis), NKX-2586 (cis) and NKX-2677. The difference with the corresponding (trans) structures is represented in Fig. 2 for the NKX-2311 case. Black, white, red, blue and yellow atoms represent carbon, hydrogen, oxygen, nitrogen and sulfur, respectively.

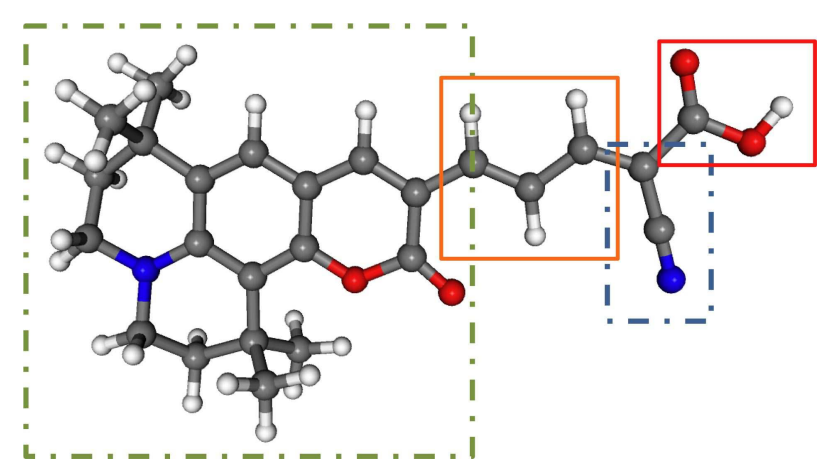

FIG. 2: (Color online) Symbolic representation of the NKX2311 (trans) coumarin dye. This structure can be compared to the NKX-2311 (cis) structure in Fig. 1(c) which differs from the orientation of the $\pi$ conjugated polymethine $(-\mathrm{HC}=\mathrm{CH}-)$ bridge (orange frame), the cyano $(-\mathrm{C}=\mathrm{N})$ (blue dot-dashed box) and the carboxylic (-COOH) (full red box) groups. The donor coumarin parent is on the left side in the green dotdashed frame. The anchoring group to the semiconducting $\mathrm{TiO}_{2}$ surface is the carboxylic unit.

$G W /$ BSE formalism yields the low-lying charge-transfer excitation energies in excellent agreement with optimized (parametrized) range-separated hybrid functionals and quantum chemistry CC2 coupled-cluster calculations with a mean absolute error smaller than $0.06 \mathrm{eV}$. Similarly, the associated oscillator strengths are found to agree very well with ajusted long-range corrected functional calculations. These results consolidate the few recent evidences showing that $G W / \mathrm{BSE}$ calculations can describe charge-transfer excitations in gas phase organic systems with an accuracy of the order of a tenth of an $\mathrm{eV}$ without any adjustable parameter.

\section{TECHNICAL DETAILS}

Our calculations are performed with the FIESTA package $\underline{28,32}-34$ which exploits atom-centered auxiliary Gaussian-basis for developing the needed two-body operators such as the dynamical ( $\omega$-frequency dependent) susceptibilities, the screened Coulomb potential $W\left(\mathbf{r}, \mathbf{r}^{\prime} ; \omega\right)$, and the self-energy in the $G W$ approximation:

$\Sigma^{G W}\left(\mathbf{r}, \mathbf{r}^{\prime} ; \omega\right)=\frac{i}{2 \pi} \int d \omega^{\prime} e^{i \omega^{\prime} 0^{+}} G\left(\mathbf{r}, \mathbf{r}^{\prime} ; \omega+\omega^{\prime}\right) W\left(\mathbf{r}, \mathbf{r}^{\prime} ; \omega^{\prime}\right)$,

where $G$ is the time-ordered one-particle Green's function. The self-energy is non-local in space and time (energy-dependent) and accounts for exchange and correlation in the present formalism. Our auxiliary basis contains six $\exp \left(-\alpha \mathbf{r}^{2}\right)$ Gaussians for the radial part of each $(s, p, d)$-channel, with an even tempered ${ }^{35}$ distribution of the localization coefficients $\alpha$ ranging from 0.1 to 3.2 a.u. except for hydrogen where the range is set to 0.1 
a.u. to 1.5 a.u. As such, our auxiliary basis contains typically 54 orbitals per atom. Such a basis derives from previous studies $28,32,33$ but with additional diffuse orbitals. Convergency tests can be found in Ref. 32 with more details about the present $G W$ implementation which is similar to that of Rohlfing and coworkers ${ }^{36.37}$ We however go beyond the plasmon-pole approximation for obtaining the dynamical correlations by using contour deformation techniques, $18,32,38$

The single-particle states needed to build the starting screened-Coulomb potential $W$ and Green's function $G$ are the Kohn-Sham DFT eigenstates in the local density approximation (LDA) as provided by the SIESTA package 39 with a large triple-zeta plus double polarization basis (TZDP) for the valence orbitals $\stackrel{40}{4}$ Using such running parameters, comparison with $G W / \mathrm{BSE}$ calculations performed with a reference planewave code ${ }^{41}$ showed excellent agreement in the case of acene/tetracyanoethylene (TCNE) donor/acceptor complexes,$\stackrel{28}{r}$ demonstrating the accuracy of the present Gaussian-based formalism. For sake of illustration, the Kohn-Sham and auxiliary basis associated with the NKX-2677 molecule (67 atoms) contain 1110 and 3618 orbitals, respectively. Our structures are relaxed at the all-electron B3LYP 6-311G(d,p) level. $\stackrel{42}{ }$

We start with a $G W$ calculation designed to obtain the correct quasiparticle spectrum, namely the correct occupied and unoccupied single-particle energy levels. As observed in several studies on gas phase (isolated) molecular systems, $, 32,33,43,45-47$ the standard "single-shot" $G_{0} W_{0}(\mathrm{LDA})$ scheme tends to produce too small energy gaps between the highest occupied (HOMO) and the lowest unoccupied (LUMO) molecular orbitals. In the case of CT excitations with a large weight on the HOMO-LUMO transition, this has been shown to result in $G_{0} W_{0} / \mathrm{BSE}$ excitation energies too small as compared to experiment, with a mean absolute error of about $0.7 \mathrm{eV}$ in the case of the small TCNE/acenes complexes: $^{28}$ Such a problem has been largely cured by several groups $28,29,32,45-47$ thanks to a partial selfconsistent cycle where the quasiparticle energies are reinjected to build updated time-ordered Green's function $G$ and screened Coulomb potential $W$, while keeping the eigenstates (Kohn-Sham one particle orbitals) frozen. $\underline{44}$ This simple self-consistent loop allows to obtain much

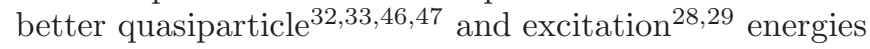
as compared to experiment. Further, the observed sensitivity of the $G_{0} W_{0}$ results to the choice of the starting zeroth-order eigenstates $28,32,44,46-49$ is significantly reduced 50 It is such an approach that we adopt in the present study.

In a second step, the neutral (optical) excitation spectrum is constructed by using the Bethe-Salpeter equations (BSE) ${ }^{22-26}$ that account for the electron-hole (excitonic) interactions. Deriving from Green's function many-body perturbation theory, the Bethe-Salpeter Hamiltonian $H^{B S E}$ is most commonly written in the two-body product basis $\phi_{i}(\mathbf{r}) \phi_{j}\left(\mathbf{r}^{\prime}\right)$ of the Kohn-Sham orbitals,,$\frac{54}{4}$ resulting in the following generalized eigenvalue problem:

$$
\left(\begin{array}{cc}
A_{v c, v^{\prime} c^{\prime}} & C_{v c, c^{\prime} v^{\prime}} \\
-C_{c v, v^{\prime} c^{\prime}}^{*} & -A_{c v, c^{\prime} v^{\prime}}^{*}
\end{array}\right)\left(\begin{array}{c}
X_{v^{\prime} c^{\prime}} \\
Y_{c^{\prime} v^{\prime}}
\end{array}\right)=\Omega\left(\begin{array}{c}
X_{v c} \\
Y_{c v}
\end{array}\right)
$$

The first diagonal block: $A^{B S E}=\left(H^{\text {diag }}+H^{\text {direct }}+\right.$ $\left.H^{\text {exch }}\right)$, accounts for resonant transitions from occupied (indexes $\mathrm{v}, \mathrm{v}^{\prime}$ ) to unoccupied (indexes c,c') eigenstates, with the following contributions:

$$
\begin{aligned}
H_{v c, v^{\prime} c^{\prime}}^{\text {diag }} & =\left(\varepsilon_{c}^{G W}-\varepsilon_{v}^{G W}\right) \delta_{v v^{\prime}} \delta_{c c^{\prime}}, \\
H_{v c, v^{\prime} c^{\prime}}^{\text {direct }} & =-\int d \mathbf{r} d \mathbf{r}^{\prime} \phi_{c}(\mathbf{r}) \phi_{v}\left(\mathbf{r}^{\prime}\right) W\left(\mathbf{r}, \mathbf{r}^{\prime}\right) \phi_{c^{\prime}}(\mathbf{r}) \phi_{v^{\prime}}\left(\mathbf{r}^{\prime}\right), \\
H_{v c, v^{\prime} c^{\prime}}^{e x c h} & =2 \int d \mathbf{r} d \mathbf{r}^{\prime} \phi_{c}(\mathbf{r}) \phi_{v}(\mathbf{r}) v\left(\mathbf{r}, \mathbf{r}^{\prime}\right) \phi_{c^{\prime}}\left(\mathbf{r}^{\prime}\right) \phi_{v^{\prime}}\left(\mathbf{r}^{\prime}\right) .
\end{aligned}
$$

The second diagonal block $\left(-A^{*}\right)$ accounts for nonresonant transitions from unoccupied to occupied levels. The off-diagonal $\mathrm{C}$ term couples resonant and nonresonant transitions, with $\mathrm{C}$ also composed of a direct and an exchange term which can be obtained from their A-block analogs by switching the (v',c') indexes. While all empty states are included in the construction of the independent-electron susceptibilities and Green's function needed for the $G W$ calculations, the number of empty states included in the BSE Hamiltonian has been set to 160. Convergency tests for the largest NKX-2677 molecule indicate that increasing this number to 200 decreases the excitation energy by less than $10 \mathrm{meV}$.

In what follows, we go beyond the Tamm-Dancoff approximation (TDA) by mixing resonant and antiresonant contributions. Namely, we do calculate the coupling $(\mathrm{C})$ and $\left(-C^{*}\right)$ blocks. As shown in recent studies,$\underline{37,55}$ the TDA tends to overestimate by a few tenths of an eV the excitations energies in small size systems where single-particle and collective excitations can strongly mix. This is what we observe here with the lowlying excitation energies which are blue-shifted by up to $0.25 \mathrm{eV}$ for the C343 dye, and $0.18 \mathrm{eV}$ in the NKX-2677 case, when the off-diagonal coupling blocks are neglected.

\section{RESULTS AND ANALYSIS}

Our results are compiled in Table 1 and in Fig. 3 with a comparison to previously published TDDFT and CC2 calculations. As expected, the TD-LDA and TD-PBE values from Ref. 10 evidence an underestimation of the transition energies related to the CT character of the excitations. The mean absolute error (MAE) averaged over the available data points amounts to $0.47 \mathrm{eV}$ and $0.44 \mathrm{eV}$ as compared to CC2 within LDA and PBE, respectively. Further, the oscillator strength seems to be significantly 


\begin{tabular}{|c|c|c|c|c|c|c|}
\hline & $\mathrm{LDA} / \mathrm{PBE}^{(a)}$ & $\mathrm{B} 3 \mathrm{LYP}^{(b)}$ & LC-BLYP ${ }^{(c)}$ & $\mathrm{BNL}^{(d)} \mathrm{J} 1 / \mathrm{J} 2$ & $\mathrm{CC} 2^{(b)}$ & $G W-\mathrm{BSE}$ \\
\hline C343 & $2.96 / 3.0(0.36 /)$ & $3.32(0.60)$ & $3.36(0.57)$ & $3.5 / 3.4(0.7 / 0.6)$ & $3.44(0.74)$ & $3.44(0.57)$ \\
\hline NKX-2388 s-trans & & $2.90(0.94)$ & $3.01(0.88)$ & $3.1 / 2.9(1.0 / 0.9)$ & $2.99(1.06)$ & $3.04(0.88)$ \\
\hline NKX-2388 s-cis & & $2.78(0.87)$ & $2.85(0.80)$ & $2.9 / 2.8(0.9 / 0.9)$ & $2.80(1.00)$ & $2.85(0.80)$ \\
\hline NKX-2311 s-trans & & $2.70(1.35)$ & $2.91(1.34)$ & $2.9 / 2.8(1.6 / 1.5)$ & $2.89(1.51)$ & $2.88(1.37)$ \\
\hline NKX-2311 s-cis & $2.35 / 2.35(1.05 /)$ & $2.56(1.19)$ & $2.73(1.12)$ & $2.7 / 2.6(1.3 / 1.2)$ & $2.71(1.33)$ & $2.67(1.13)$ \\
\hline NKX-2586 s-trans & & $2.50(1.71)$ & $2.81(1.83)$ & $2.8 / 2.6(2.1 / 2.0)$ & $2.81(2.01)$ & $2.74(1.88)$ \\
\hline NKX-2586 s-cis & $2.10 / 2.15(1.23 /)$ & $2.40(1.55)$ & $2.66(1.52)$ & $2.6 / 2.5(1.7 / 1.7)$ & $2.66(1.74)$ & $2.57(1.59)$ \\
\hline NKX-2677 & & $2.23(1.49)$ & $2.67(1.76)$ & $2.7 / 2.5(2.0 / 1.8)$ & $2.71(2.17)$ & $2.56(1.69)$ \\
\hline
\end{tabular}

TABLE I: Calculated lowest $\left(S_{0} \rightarrow S_{1}\right)$ singlet transition energies (in eV). The $G W$-BSE results calculated in the present study are compared to the TD-LDA, TD-PBE, TD-B3LYP, TD-LC-BLYP, TD-BNL and CC2 calculations from Refs. 10 13. We provide the B3LYP results from Ref. 11 which are in excellent agreement with the results of Ref. 12 . The numbers in parenthesis indicate the associated oscillator strengths.

${ }^{a}$ Ref. 10 .

${ }^{b}$ Ref. 11 .

${ }^{c}$ Ref. 12 .

${ }^{d}$ Ref. 13 .

underestimated, in particular, surprisingly, in the case of the smaller C343 parent molecule expected to show the smallest CT character.

Thanks to its $20 \%$ of exact exchange, the B3LYP results of Ref. 11 (filled black circles in Fig. 3) indicate a reduced MAE of $0.2 \mathrm{eV}$. However, the discrepancy can still be as large as $0.48 \mathrm{eV}$ for the NKX-2677 structure. This is certainly the signature that in the long-range charge-separation limit, the restricted amount of exact exchange in the B3LYP functiona ${ }^{56}$ is not enough to account for the correct electron-hole interaction. To illustrate that point, we plot in Fig. 4 the Kohn-Sham (a,d) HOMO and (b,e) LUMO eigenstates associated with the C343 and NKX-2677 structures. While the HOMO states are found to be rather delocalized, the LUMO in the NKX-2677 dye is clearly much more localized close to the electron-acceptor cyanoacrylic group, resulting in an enhanced CT character as compared to the C343 parent molecule.

The nature of the transitions can be better quantified by studying the electron and hole spatial localization in the excited states. This can be achieved by taking the expectation value of the electron/hole position operator $\delta\left(\mathbf{r}-\mathbf{r}_{e} / \mathbf{r}_{h}\right)$ over the two-body $\psi\left(\mathbf{r}_{e}, \mathbf{r}_{h}\right)$ BSE excitonic wavefunction, leading to an electron/hole probability of presence averaged over the hole/electron position. The resulting densities are provided in Fig. 4(c,f) with an isocontour representation for the C343 and NKX-2677 molecules. These densities allow to obtain the mean electron/hole positions (see red arrows in Fig. 4) and the related average electron-hole separation distance which amounts to $3.2 \AA$ in C343. This clear CT character is certainly at the origin of the difficulties met by LDA or $\mathrm{PBE}$ to describe such an excitation. In the NKX-2677 case, this average distance increases to $4.6 \AA$ as a signature of the enhanced CT character, explaining that the B3LYP results significantly worsen from C343 to NKX2677.

We now come to the central results of the present study, namely the many-body perturbation theory data.
In contrast with the LDA, PBE or even B3LYP results, our $G W$-BSE values (empty red squares in Fig. 3) are in much better agreement with the $\mathrm{CC} 2$ data points, with a mean absolute error of $0.06 \mathrm{eV}$. Such an agreement is remarkable accounting for the fact that the present $G W /$ BSE approach does not contain any adjustable parameter. Concerning the longest NKX-2677 dye, which shows the largest discrepancy with $\mathrm{CC} 2$ calculations, we observe that our result falls within the values provided by the range-separated hybrid ${ }^{57}$ (RSH) BNL functional study $\underline{13}$ where two different strategies to optimize $a b$ initio (non-empirically) the range-separation parameter have been tested 58 As compared to the RSH-BNL study, our $G W$-BSE results differ by a MAE ranging from 0.04 $\mathrm{eV}$ to $0.07 \mathrm{eV}$, that is well within $0.1 \mathrm{eV}$. As emphasized in Ref. 13, the CC2 approach is also not free from approximations ${ }^{59}$ and differences of the order of $0.1 \mathrm{eV}$ as compared to more accurate e.g. CASPT2 calculations are certainly to be expected.

Clearly, as compiled in Table [1 TDDFT calculations with the LC-BLYP functional ${ }^{60}$ also provide excellent results,,$\underline{12}$ with a $0.03 \mathrm{eV}$ MAE as compared to $\mathrm{CC} 2$, smaller than our $G W$-BSE $0.06 \mathrm{eV}$ MAE value. However, as emphasized in Ref. 12, the range-separation parameter $\mu$ in the LC-BLYP study has been precisely adjusted to minimize the root mean square error with $\mathrm{CC} 2$ calculations. The best-fit $\mu$ value for these systems ( $\mu=0.17$ a.u.) is found to be much smaller than the original $\mu=0.33$ advocated by Iikura and coorkers $\frac{60}{}$. The strong dependence of the excitation energies as a function of $\mu$ indicates that the choice of the originally recommended $\mu=0.33$ value would lead to a significant overestimation of the transition energies (by as much as $0.3-0.4 \mathrm{eV}$, see Fig. 4 of Ref.12). This leads to the standard question of the choice and transferability of the range-separation parameter(s). One observes however that with the best-fit $\mu$ value, the correlation between LC-BLYP and CC2 results is very remarkable, showing that this class of systems can be described by a unique parameter.

Bearing important consequences on the use of such 


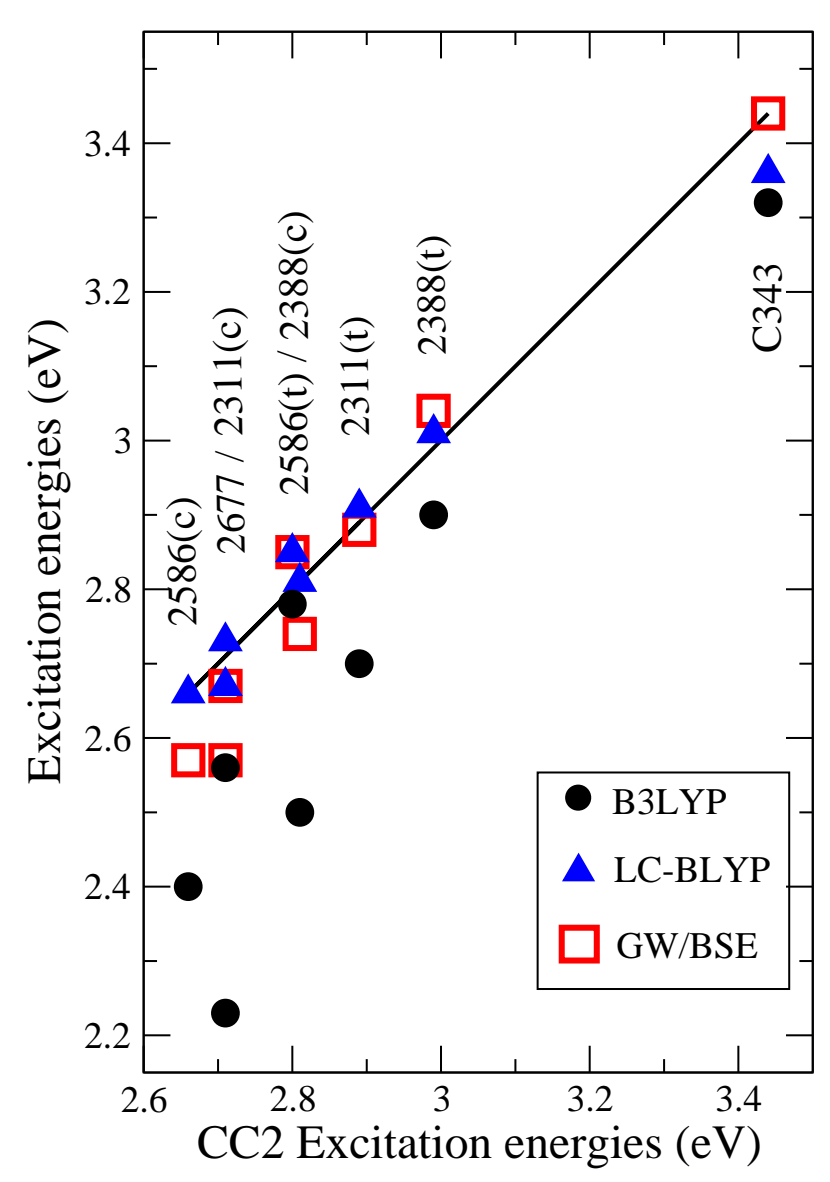

FIG. 3: (Color online) Calculated lowest singlet excitation energies (in $\mathrm{eV}$ ) as a function of the coupled-cluster $\mathrm{CC} 2$ reference values. Results in perfect agreement with the $\mathrm{CC} 2$ calculations should fall on the first diagonal (black line). The present $G W$-BSE calculations (empty red squares) are compared to the TD-B3LYP results of Refs. 11, 12 (filled black circles) and the "adjusted" (see text) TD-LC-BLYP data from Ref. 12 (blue triangles up). The coumarins name are indicated by their number (removing the NKX prefix) with (c) standing for -cis and (t) for -trans. The axes physical length is scaled according to their respective energy range.

dyes in DSSCs, our $G W$-BSE calculations confirm the range-separated hybrid TDDFT and $\mathrm{CC} 2$ data leading to the conclusion that the onset of absorption is significantly red shifted with increasing size length. This evolution is in clear contrast with the behavior of CT excitations in well separated gas phase donor/acceptor dyads where the exciton binding energy scales as the inverse distance between the two molecules, leading to an increase of the absorption energy onset. However, contrary to well separated donor/acceptor systems, the quasiparticle HOMO-LUMO gap in donor/acceptor dyads connected by a conducting $\pi$-conjugated bridge does not remain constant with varying bridge length. This is clearly exemplified in Table $\Pi$ where the $G W$ HOMO-LUMO gap is found to quickly decrease from the C343 molecule to
C343

a)

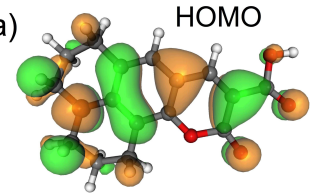

b)

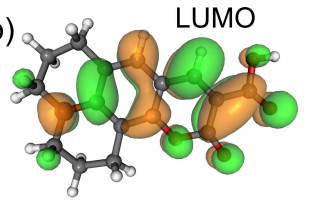

c)

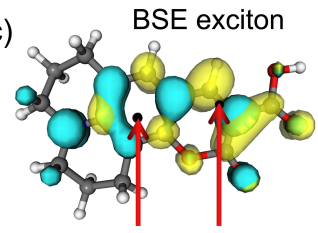

Nkx2677

d)

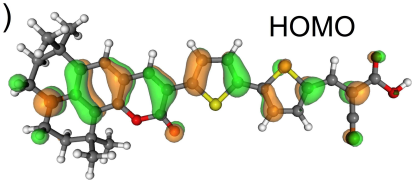

e)

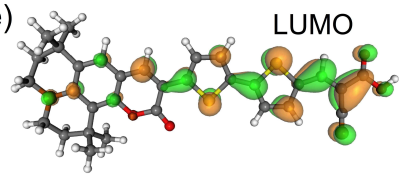

f)

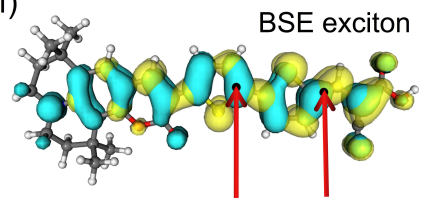

FIG. 4: (Color online) (a) and (b) Isocontour representation of the C343 HOMO and LUMO Kohn-Sham eigenstates. Different colors indicate different signs of the wavefunction. In (d) and (e), similar plots for NKX-2677. In (c) and (f), isocontour representation of the electron (yellow) and hole (light blue) probability distribution for the lowest C343 and NKX2677 singlet excited states, respectively, as obtained within BSE. The red arrows indicate the average hole (left arrow) and electron (right arrow) positions. The C343 and NKX2677 molecules are not represented on the same scale.

the longest NKX-2677 dye. Except for the large variation of the electronic affinity (EA) from the C343 parent to the NKX-2388 system, analysis of the $G W$ HOMO and LUMO quasiparticle energies indicates that this gap reduction stems both from a destabilisation of the HOMO (decrease of the ionization potential IP) and a stabilization of the LUMO (increase of the EA). Subtracting the $G W$ HOMO-LUMO gap from the singlet $G W$-BSE excitation energy, one finds that the electron-hole (excitonic) binding energy $E_{B}$ decreases with donor-acceptor distance, but this effect is not strong enough to counterbalance the decrease of the HOMO-LUMO gap. This is an important feature which explains that the longest NKX-2677 dye is most efficient in harvesting photons in the visible range.

We close this study by commenting on the oscillator strength associated with the calculated transitions (see the numbers in parenthesis in Table $\prod$ and Fig. (5). Our $G W$-BSE values (red empty squares) lead to an excellent agreement with the parametrized LC-BLYP (blue up triangles) calculations, with a perfect match for the C343 and NKX-2388 molecules. Such an agreement is remarkable given the fact that the two approaches are very different. Both $G W$-BSE and LC-BLYP provide 


\begin{tabular}{l|llll}
\hline & IP & EA & Gap & $E_{B}$ \\
\hline C343 & 7.21 & 0.60 & 6.61 & 3.17 \\
NKX-2388 s-trans & 7.12 & 1.33 & 5.79 & 2.75 \\
NKX-2388 s-cis & 7.11 & 1.50 & 5.61 & 2.76 \\
NKX-2311 s-trans & 6.93 & 1.55 & 5.38 & 2.50 \\
NKX-2311 s-cis & 6.92 & 1.71 & 5.21 & 2.54 \\
NKX-2586 s-trans & 6.76 & 1.70 & 5.06 & 2.32 \\
NKX-2586 s-cis & 6.77 & 1.85 & 4.91 & 2.34 \\
NKX-2677 & 6.52 & 1.62 & 4.90 & 2.33 \\
\hline
\end{tabular}

TABLE II: Calculated $G W$ ionization potential (IP), electronic affinity (EA), and HOMO-LUMO gap. The exciton binding energy $E_{B}$ is the $G W$ HOMO-LUMO gap minus the $\mathrm{BSE}$ (singlet) excitation energy. Energies are in eV.

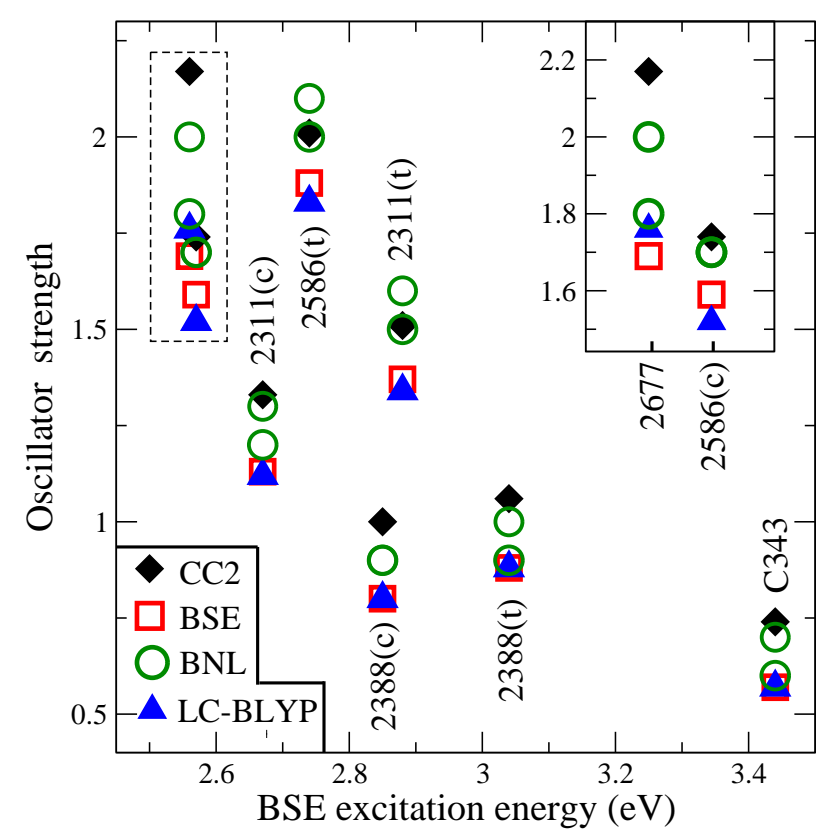

FIG. 5: (Color online) Theoretical strength as a function of the $G W$-BSE excitation energies (in eV). The BSE values (red empty squares) are compared to the CC2 (black diamond), LC-BLYP (blue triangle up), and the BNL J1/J2 (empty green circles) results. Results for the NKX-2677 and NKX-2586 (s-cis) in the dashed box are reproduced in the up-right inset.

lower values as compared to $\mathrm{CC} 2$ calculations, the $\mathrm{BNL}$ data yielding somehow intermediate results. The largest discrepancies occur for the NKX-2677 molecule, with a $22 \%$ difference between the $G W$-BSE and CC2 values. For this system, $G W$-BSE and LC-BLYP agree again extremely well, within $4 \%$, while the two BNL values (see Inset) show their largest spread, indicating that the oscillator strength for this structure is very sensitive to the chosen formalism and related parameters. Further anal- ysis is needed to understand these variations from one type of calculation to another. Beyond differences, one should emphasize that all studies agree on the fact that the NKX-2677 dye presents one of the largest oscillator strength, yet another factor explaining that it leads to one of the largest conversion efficiency in the coumarin family.

\section{CONCLUSION}

In conclusion, we have studied within the many-body Green's function $G W$ and BSE perturbation theory the excitation energies of a family of coumarin dyes recently shown to be very promising candidates for replacing ruthenium-based chromophores in dye-sensitized solar cells (DSSC). In such donor-bridge-acceptor molecules, the lowest singlet excitations are characterized by a charge-transfer character that varies with the length of the $\pi$-conjugated bridge. As a result, TD-B3LYP calculations can lead to an error as large as $\sim 0.5 \mathrm{eV}$ as compared to reference quantum chemistry coupled-cluster CC2 calculations, despite the $20 \%$ of exact exchange contained in its functional form. We demonstrate that the $G W /$ BSE approach leads to an excellent agreement with $\mathrm{CC} 2$ data with a mean absolute error of the order of $0.06 \mathrm{eV}$ for the excitation energies. Such an accuracy is comparable to the best results provided by TDDFT calculations with optimized long-range corrected rangeseparated hybrids, but with a parameter-free approach that performs equally well for extended insulating or metallic systems and gas phase organic molecules. Such an excellent agreement is also demonstrated for the related oscillator strengths. The ability of the $G W / \mathrm{BSE}$ approach to describe both Frenkel and charge-transfer excitations in finite size molecular systems or extended semiconductors originates in particular from the use of the screened Coulomb potential $W$ that automatically adjusts the strength and range of the Coulomb interactions. This flexibility may proove as a significant advantage in the study of DSSCs where both the organic dye and the extended $\mathrm{TiO}_{2}$ semiconductor must be treated with sufficient accuracy.

Acknowledgements. I.D. acknowledges funding from the CEA "Eurotalent" program and C.F. a joint CEA/CNRS BDI fellowship. The authors are indebted to L. Kronik for sending the initial geometries of the coumarins studied in this work, and to C. Attaccalite and $\mathrm{V}$. Olevano for many suggestions and critical readings of our manuscript. Calculations have been performed thanks to French national supercomputing IDRIS center at Orsay under contract $\mathrm{n}^{o} \mathrm{i} 2012096655$. 
son, Chem. Rev. 110, 6595-6663 (2010).

3 K. Hara, T. Sato, R. Katoh, A. Furube et al. J. Phys. Chem. B 107, 597-606 (2003).

4 J. M. Rhem, G. L. McLendon, Y. Nagasawa, K. Yoshihara, J. Moser and M. Grätzel, J. Phys. Chem. 100, 9577 (1996).

${ }^{5}$ K. Hara, M. Kurashige, Y. Dan-oh, C. Kasada, A. Shinpo, S. Suga, K. Sayama and H. Arakawa, New J. Chem. 27, 783-785 (2003).

${ }^{6}$ E. Runge and E.K.U. Gross, Phys. Rev. Lett. 52, 997 (1984); M.A.L. Marques, C.A. Ullrich, F. Nogueira, A. Rubio, K. Burke, and E.K.U. Gross (eds.), Time-Dependent Density Functional Theory (Springer-Verlag, 2006); Mark E. Casida, J. Mol. Struct. (Theochem) 914, 3 (2009).

7 A. Dreuw, M. Head-Gordon, J. Am. Chem. Soc. 126, 40074016 (2004).

8 Similar problems arise in extended solids for Wannier excitons with large effective bohr radius characterized by a large average electron-hole distance. See: S. Botti, F. Sottile, N. Vast, V. Olevano, L. Reining, H.-C. Weissker, A. Rubio, G. Onida, R. Del Sole, and R. W. Godby, Phys. Rev. B 69, 155112 (2004).

9 An interesting "static" analog, illustrating the role of nonlocal exchange for long-range electron-hole interaction, is the image-charge potential problem within DFT. For an analysis in the case of metallic surfaces, see: I.D. White, R.W. Godby, M.M. Rieger, and R.J. Needs, Phys. Rev. Lett. 80, 4265 (1998)

10 R. Sánchez-de-Armas, M. Ángel San Miguel, J. Oviedo and J. Fdez. Sanz, Phys. Chem. Chem. Phys. 14, 225 (2012).

11 Y. Kurashige, T. Nakajima, S. Kurashige, K. Hirao and Y. Nishikitani, J. Phys. Chem. A 111, 5544-5548 (2007).

12 B.M. Wong and J.G. Cordaro, J. Chem. Phys. 129, 214703 (2008).

13 T. Stein, L. Kronik and R. Baer, J. Chem. Phys. 131, 244119 (2009).

14 N.S. Sariciftci, L. Smilowitz, A.J. Heeger, F. Wudl, Science 258, 1474 (1992); S.A. Jenekhe, J.A. Osaheni, Science 265, 765 (1994); J.L. Bredas, D. Beljonne, V. Coropceanu, J. Cornil, Chem. Rev. 104, 4971 (2004); Y. Kanai and J.C. Grossman, Nano Lett. 8, 3049 (2004).

15 L. Hedin, Phys. Rev. 139, A796 (1965).

16 G. Strinati, H.J. Mattausch, W. Hanke, Phys. Rev. Lett. 45, 290 (1980); ibid, Phys. Rev. B 25, 2867 (1982).

17 M.S. Hybertsen, S.G. Louie, Phys. Rev. B 34, 5390 (1986).

18 R.W. Godby, M. Schlüter, and L.J. Sham, Phys. Rev. B 37, 10159 (1988).

19 G. Strinati, Rivista dek Nuovo Cimento 11, 1-86 (1988).

${ }^{20}$ G. Onida, L. Reining, A. Rubio, Rev. Mod. Phys. 74, 601 (2002).

21 For a compilation of $G W$ results in the crystalline solid phase, see e.g. W.G. Aulbur, L. Jonsson, J.W. Wilkins, in Solid State Physics, edited by H. Ehrenreich (Academic, Orlando, 1999), Vol. 54, p. 1.

22 L.J. Sham and T.M. Rice, Phys. Rev. 144, 708 (1966); W. Hanke and L.J. Sham, Phys. Rev. Lett. 43, 387 (1979).

23 G. Strinati, Phys. Rev. Lett. 49, 1519 (1982); H. J. Mattausch, W. Hanke, and G. Strinati, Phys. Rev. B 27, 3735 (1983); Phys. Rev. B 29, 5718 (1984).

24 M. Rohlfing, S.G. Louie, Phys. Rev. Lett. 80, 3320 (1998)

25 L.X. Benedict, E. Shirley, R.B. Bohn, Phys. Rev. Lett. 80, 4514 (1998).

${ }^{26}$ S. Albrecht, L. Reining, R. Del Sole, G. Onida, Phys. Rev. Lett. 80, 4510 (1998).
27 J.M. Garcia-Lastra, K.S. Thygesen, Phys. Rev. Lett. 106, 187402 (2011).

28 X. Blase, C. Attaccalite, Appl. Phys. Lett. 99, 171909 (2011).

29 Björn Baumeier, Denis Andrienko, and Michael Rohlfing, J. Chem. Theory Comput., Article ASAP (2012).

30 I.J. Hanazaki, Phys. Chem. 76, 1982 (1972).

31 D. Rocca, D.Y. Lu, and G. Galli, J. Chem. Phys. 133, 164109 (2010).

32 X. Blase, C. Attaccalite, V. Olevano, Phys. Rev. B 83, 115103 (2011).

33 C. Faber, C. Attaccalite, V. Olevano, E. Runge, X. Blase, Phys. Rev. B 83, 115123 (2011).

34 C. Faber, J. Laflamme Janssen, M. Côté, E. Runge, and X. Blase, Phys. Rev. B 84, 155104 (2011).

35 I. Cherkes, S. Klaiman, and N. Miseyev, Int. J. Quantum Chem. 109, 2996 (2009); and references therein.

36 M. Rohlfing, P. Krüger, J. Pollmann, Phys. Rev. B 52, 1905 (1995).

37 Y. Ma, M. Rohlfing, C. Molteni, Phys. Rev. B 24, 241405 (2009); ibid., J. Chem. Theory Comput. 6, 257-265 (2010); and references therein.

38 B. Farid, in Electron Correlation in the Solid State, edited by N. H. March (World Scientific, Singapore, 1999), p. 217, and references therein.

39 J.M. Soler, E. Artacho, J.D. Gale, A. García, J. Junquera, P. Ordejón and D. Sánchez-Portal, J. Phys.: Condens. Mater 14, 2745-2779 (2002). The Siesta confinement criteria on basis orbitals is released by imposing a very large cut-off radius ( $\sim 12$ a.u.)

40 We use standard norm-conserving pseudopotentials. See: N. Troullier and J.-L. Martins, Phys. Rev. B 43, 1993 (1991).

41 A. Marini, C. Hogan, M. Grüning, D. Varsano, Comput. Phys. Commun. 180, 1392 (2009).

42 M. J. Frisch et al., GAUSSIAN 09, Revision B.01, Gaussian, Inc., Wallingford CT 2010.

43 P.H. Hahn, W.G. Schmidt and F. Bechstedt, Phys. Rev. B 72, 245425 (2005). In this study, the importance of correcting the starting eigenvalues in the construction of $W$ is emphasized for molecular systems, bringing some light in the discussions concerning the update of the eigenvalues in the Green's function only (the $G W_{0}$ scheme) or in both $G$ and $W$. See also Ref. 44 for extended solids.

44 Weidong Luo, Sohrab Ismail-Beigi, Marvin L. Cohen, and Steven G. Louie, Phys. Rev. B 66, 195215 (2002); M. Shishkin and G. Kresse, Phys. Rev. B 75, 235102 (2007); and references therein.

${ }^{45}$ L. Tiago, P. R. C. Kent, R. Q. Hood, and F. A. Reboredo, J. Chem. Phys. 129, 084311 (2008).

46 S. Sharifzadeh, A. Biller, L. Kronik, J.B. Neaton, Phys. Rev. B 85, 125307 (2012).

47 S. Refaely-Abramson, S. Sharifzadeh, N. Govind, J.B. Neaton, R. Baer, L. Kronik, arXiv:1203.2357.

48 N. Marom, X. Ren, J.E. Moussa, J. R. Chelikowsky and L. Kronik, Phys. Rev. B 84, 195143 (2011).

49 T. Körzdörfer, N. Marom, Phys. Rev. B 86, 041110(R) (2012).

50 As shown in Ref. 32 for a large family of organic molecules, this partial self-consistency scheme leads to nearly identical quasiparticle HOMO-LUMO gaps starting either from DFT-LDA or HF-like eigenvalues, as a clear signature of the reduced sensitivity to the starting mean-field approximation. The $G_{0} W_{0}(\mathrm{HF})$ results were shown to be much 
closer to the self-consistent $G W$ results than $G_{0} W_{0}$ (LDA). Similar trends have been observed recently with fully selfconsistent $G W$ calculations on isolated atoms or small molecules for which such calculations are feasible ${ }^{51-53}$ This conclusion is expected to hold only for small systems with reduced screening. For hybrid systems such as Grätzel cells, with molecular dyes deposited on semiconducting surfaces, neither the $G_{0} W_{0}(\mathrm{LDA})$ nor the $G_{0} W_{0}(\mathrm{HF})$ approximation are expected to be reliable for both the donor (dye) and the acceptor $\left(\mathrm{TiO}_{2}\right)$, emphasizing the importance of self-consistency in some form, or the use of hybrid functionals $\frac{48,49}{4}$ that may perform well for both systems.

51 C. Rostgaard, K. W. Jacobsen, and K. S. Thygesen, Phys. Rev. B 81, 085103 (2010).

52 San-Huang Ke, Phys. Rev. B 84, 205415 (2011).

53 Fabien Bruneval, J. Chem. Phys. 136, 194107 (2012).

54 For isolated systems, wavefunctions can be taken to be real and we do not consider here the complex conjugated eigenstates.

55 M. Grüning, A. Marini, X. Gonze, Nano Lett. 9, 2820 (2009).

56 A.D. Becke, J. Chem. Phys. 98, 5648 (1993).
57 A. Savin, in Recent Developments and Applications of Modern Density Functional Theory, edited by J. M. Seminario bibitemElsevier, Amsterdam, Chap. 9, pp. 327-354 (1996); T. Leininger, H. Stoll, H. J. Werner, and A. Savin, Chem. Phys. Lett. 275, 151 (1997); J. Toulouse, F. Colonna, and A. Savin, Phys. Rev. A 70, 062505 (2004).

58 In the BNL approach, the range separation parameter is obtained $a b$ initio by minimizing the MAE between the Kohn-Sham HOMO and/or LUMO eigenvalues and the corresponding quantities obtained with a much more accurate total energy difference $\triangle \mathrm{SCF}$ approach for the neutral and charged systems. Depending on the chosen criteria (optimization of the HOMO only of the neutral and charged systems, or of the HOMO and LUMO of the neutral system, the so-called "J1" and "J2" schemes), slightly different values can be obtained. See Ref. 13 .

59 It is to be observed that due to computational costs at the time of publication, the CC2 calculations were performed with a limited SV(P) basis. See Ref. 11.

${ }^{60}$ H. Iikura, T. Tsuneda, T. Yanai, and K. Hirao, J. Chem. Phys. 115, 3540 (2001). 